\title{
Real time quantification of Monte Carlo steps for different time scales
}

\author{
R. Smirnov-Rueda ${ }^{a}$ \\ Computational Magnetism Group, School of Electronic Engineering \& Computer Systems, \\ University of Wales, Dean Street, Bangor, LL57 1UT, United Kingdom \\ O. Chubykalo \\ IBM Almaden Research Center, Magnetic Theory and Modeling, 650 Harry Road, San Jose, \\ California, 95120
}

U. Nowak

Theoretische Tieftemperaturphysik, Gerhard-Mercator University of Duisburg, 47048 Duisburg, Germany

\author{
R. W. Chantrell \\ Computational Magnetism Group, School of Electronic Engineering \& Computer Systems, \\ University of Wales, Dean Street, Bangor, LL57 1UT, United Kingdom
}

\section{J. M. Gonzalez}

Instituto de Ciencia de Materiales de Madrid, CSIC, Cantoblanco, 28049 Madrid, Spain

\begin{abstract}
Time quantification of Monte Carlo steps is studied by the implementation of a new technique which takes into account the realistic size of thermal fluctuations of magnetization along with LandauLifshitz-Gilbert dynamic correlations. The computational model has been specifically developed for an ensemble of isolated single-domain particles. The numerical results have been compared with Langevin dynamics calculations and theoretically predicted Brown's asymptotes for relaxation time of single spin system. In addition we demonstrated that real time quantification of Monte Carlo steps is also possible for different time scales. Implementation of real time scales into Monte Carlo calculations for different sizes of time steps is shown to be convergent to the expected value if the Monte Carlo acceptance rate is taken into account.
\end{abstract}

\section{INTRODUCTION}

The understanding of slow dynamic behavior in extended systems of many interacting magnetic moments over large time intervals has considerable practical implications for magnetic recording and, particularly, for evaluation of long-term stability of magnetically recorded information. The theoretical formalism for studying thermally activated magnetization reversal is based on the solution of the Landau-Lifshitz-Gilbert (LLG) equation of motion with a fluctuation term representing a random thermal force. In some special cases, a corresponding Fokker-Planck equation ${ }^{1}$ yields characteristic relaxation time values as asymptotic solutions in the large energy barrier limit. ${ }^{2}$ This provides an important foundation for real time quantification of different computational techniques such as Langevin dynamics (LD), based on the exact numerical solution to the Langevin equations and Monte Carlo (MC) method, which uses a random generation of new spin configurations to reproduce the structure of a particular stochastic process. With regard to the latter method, in its original form MC generation of system configurations is not based on the real quantification of time steps. However, despite this disadvantage, the conventional MC technique is a powerful tool for simulation of thermally activated reversal over large energy barriers. In contrast, the LD approach yields explicit dynamic

${ }^{a)}$ Electronic mail: roman@sees.bangor.ac.uk information resulting directly from the numerical solution of the LLG equation but its application is limited to short time scales up to the order of $10^{-9} \mathrm{~s}$.

Recently, we have attempted ${ }^{3}$ to provide the conventional MC scheme with explicit details on the real size of time steps along with dynamic correlations arising from the LLG equation. Comparison of the time quantified MC approaches with corresponding LD calculations showed a validity for the theoretically justified relationship between the MC steps and real time intervals. Another comparison of the numerical results for the time quantified $\mathrm{MC}$ scheme was made with analytical formula for the relaxation rate in the high damping regime.,

The work in Ref. 3 represented the first attempt to use different time scales for quantified MC steps, testing the validity of that implementation by direct comparison with LD calculations. However, it is also important to test this procedure against Brown's well-known analytical results for the relaxation time, which was predicted only for an ensemble of isolated single-domain particles. This is thought to be a necessary and important preliminary step before undertaking further realistic calculations in micromagnetic models with many degrees of freedom. On the other hand, it is expected that practical implementation of different time scales will considerably increase the effectiveness of MC calculations in comparison with the LD scheme in the limit of large energy barriers. 


\section{COMPUTATIONAL METHOD}

The computational scheme outlined in Ref. 3 comprises a modification of statistical properties of the conventional MC approach ${ }^{6}$ in order to introduce explicit and detailed information on dynamic correlations available from the linearized LLG equations with the damping term and random force. Integrated over a time interval $\Delta t$, the LLG equation for a system of coupled spins gives ${ }^{3}$

$$
m_{i}^{\alpha}(t+\Delta t)-m_{i}^{\alpha}(t)=\sum_{\beta} \sum_{j} L_{i j}^{\alpha \beta} m_{j}^{\beta} \Delta t+F_{i}^{\alpha},
$$

where $m_{i}^{\alpha}=\left(M_{i}^{\alpha}-M_{i}^{\alpha(0)}\right) / M_{s}$ is a deviation of a magnetic moment $M_{i}$ from the local equilibrium value $M_{i}^{0}$ and reduced to the value of saturation magnetization $M_{s} ; \alpha, \beta$ are Cartesian components, and $F_{i}=f_{i} \Delta t$ represents the effect of thermal fluctuations on the orientation of local magnetization.

The matrix elements $L_{i j}$ of the set of linearized and coupled LLG equations are calculated for the corresponding local equilibrium state. In the case of an ensemble of isolated noninteracting particles, the correlation term $L_{i j}$ is not necessary, leaving in the right-hand side of Eq. (1) only uncorrelated random displacements $F_{i}$. Nevertheless, in contrast to the conventional MC approach, ${ }^{6}$ the statistical properties of thermal fluctuations are driven by the covariance matrix $\mu_{i j}$,

$$
\left\langle F_{i}^{\alpha}(t+\Delta t) F_{j}^{\beta}(t)\right\rangle=\mu_{i j}^{\alpha \beta} \Delta t,
$$

defined in the LD approach by application of the fluctuationdissipation theorem: ${ }^{7}$

$$
\mu_{i j}^{\alpha \beta}=-k T\left(L_{i k}^{\alpha \gamma}\left[A_{k j}^{\gamma \beta}\right]^{-1}+L_{j k}^{\alpha \gamma}\left[A_{k i}^{\gamma \beta}\right]^{-1} .\right.
$$

An assumption on the equivalence of statistical properties between $\mathrm{MC}$ and $\mathrm{LD}$ random deviations gives rise to the implementation of a realistic time scale for one MC step. According to relationship (2), each component of particle magnetization generated randomly in the $\mathrm{MC}$ scheme has a Gaussian distribution analogous to that of LD. On the other hand, the symmetric energy matrix $A_{i j}$ is evaluated from the expansion of the micromagnetic Hamiltonian in a local equilibrium state $M_{i}^{0}$. Thus, fluctuations of magnetization determined by the matrix $\mu_{i j}$ will depend not only on system parameters such as damping, volume, temperature, etc., but also on a system configuration in the local equilibrium state. In the particular case of an ensemble of single-domain particles, expression (2) for statistical properties of fluctuating magnetization takes the following form:

$$
\mu_{i i}^{\beta \beta}=k_{B} T \alpha \Delta t\left(1-\left(M_{i}^{\beta(0)}\right)^{2}\right) / k_{E} V_{i}\left(1+\alpha^{2}\right),
$$

where $k_{B}$ is Boltzmann's constant, $T$ is the temperature, $V_{i}$ is the volume of a single-domain particle, $K_{E}$ is the easy anisotropy constant, $\Delta t$ is the time interval necessary to average the fluctuations, and $\alpha$ is the damping constant. In LD calculations $\Delta t$ plays the role of the time integration step for the numerical solution of the LLG equation.

Extension of the MC calculation scheme with the information on dynamic correlations available from LLG equations gives a further extension of the above-mentioned representation of MC time units. More specifically, it is assumed that in the spirit of the LD method, each value of
MC-generated magnetization $m_{i}(t+\Delta t)$ has to be updated for coupled spins by including dynamic correlations from Eq. (1). After this procedure the new orientation of magnetization will be accepted or rejected according to the corresponding MC criteria.

In this article we apply the presented computational technique to the micromagnetic model of an isolated singledomain particle system in order to compare relaxation times with Brown's theoretical predictions and analogous Langevin dynamics calculations.

\section{MICROMAGNETIC MODEL}

Here we considered an ensemble of isolated spins, i.e., our micromagnetic model will not include exchange and magnetostatic interactions between particles. Every singledomain particle has an easy anisotropy axis parallel to the $z$ axis. In the initial state all local magnetic moments were directed along the positive direction of the $z$ axis. An external magnetic field has been measured in terms of the anisotropy field and was applied in the negative direction of the $z$ axis.

Throughout this work we have used the following simulation parameters: high damping constant $\alpha=4$ and the value $h_{\text {app }}=0.75$ for the applied magnetic field reduced to the anisotropy field $\left(h=H_{\mathrm{APP}} / H_{A}\right)$. In order to identify an integration step $\Delta t$ in the LLG equation and the size of a trial step in MC calculations we also implemented the following specific values for the easy anisotropy constant $k_{E}=4.2$ $\times 10^{6} \mathrm{ergs} / \mathrm{cm}^{3}$ and saturation magnetization $M_{s}=1.4$ $\times 10^{3} \mathrm{emu} / \mathrm{cm}^{3}$. The modeled system consisted of 500 isolated single-domain particles.

\section{RESULTS AND DISCUSSIONS}

In our simulations using LD and the MC method we calculated the relaxation time as a function of the corresponding energy barrier which in the case of single spins " $i$ "' is defined by the normalized anisotropy and the reduced applied field:

$$
\Delta E_{i}=K_{E} V_{i}\left(1-h_{\text {app }}\right)^{2},
$$

where $V_{i}$ is the volume of particle " $i$. ."

A comparison was made with the characteristic time $\tau$ calculated asymptotically from the Fokker-Planck equation ${ }^{2}$ for high energy barriers:

$$
\tau=\tau_{0} \exp \left(\Delta E / k_{B} T\right) \text {, }
$$

where the prefactor $\tau_{0}$ is given by ${ }^{8}$

$$
\tau_{0}=\frac{\left(1+\alpha^{2}\right)}{\alpha \gamma H_{A}} \frac{\sqrt{\pi} \sqrt{T^{*}}}{\left(1-h^{2}\right)(1-h)} .
$$

Here $T^{*}$ is $k_{B} T$ normalized to the maximum attainable anisotropy energy $K_{E} V_{i}$.

In Fig. 1 we compare MC calculations and LD simulations of thermally activated reversal with the same time step. In all cases the large energy barrier dependence of relaxation time is found to be in excellent agreement with Brown's theoretical predictions. ${ }^{8}$ In view of getting a more effective computational scheme based on the MC approach, we studied the real time quantification of $\mathrm{MC}$ steps for different 


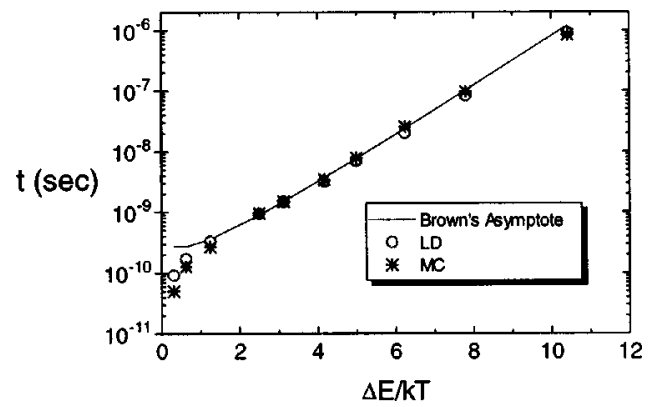

FIG. 1. Relaxation time vs energy barrier. The LD and MC simulation data are compared with Brown's asymptotic formulas for the following parameters: $\alpha=4$ and $h_{\text {app }}=0.75$.

values of time steps. The latter has been defined by the factor "scale" which represents the size of time step used in the MC approach relative to the time step in LD calculations. Large values of the scale parameter represent a significant increase in computational speed and can be used for systems with particularly large energy barriers.

In Fig. 2 we show the MC evaluation of relaxation times calculated with implementation of different time scales. Observable disagreement for larger scales is related to the fact that the MC acceptance rate decreases gradually as the size of fluctuations of magnetization is increased. For instance, in the case of scale $=10^{4}$ only one-tenth of the newly generated configurations pass through the $\mathrm{MC}$ acceptance criteria whereas for scale $=1$, the probability of acceptance is very close to 1 . These discrepancies can be reduced drastically if the total relaxation time value does not include MC moves with no modification of the system configurations.

In Fig. 3 we presented the data of relaxation time (corresponding to Fig. 2) normalized with respect to the probability of MC acceptance. As was expected, results of MC calculations for different size of time step converged to Brown's asymptote. A deviation from the theoretically predicted value is more significant for bigger scales. This error presumably arises from a breakdown of the simple scaling given by Eq. (2) for large fluctuations. Nevertheless, the agreement for large scales is still reasonably good and makes the corresponding calculation much faster. Effectiveness of the MC scheme provided for one time scale with respect to the other depends on the system parameters as well as on the range of the energy barriers. However, such a variation does

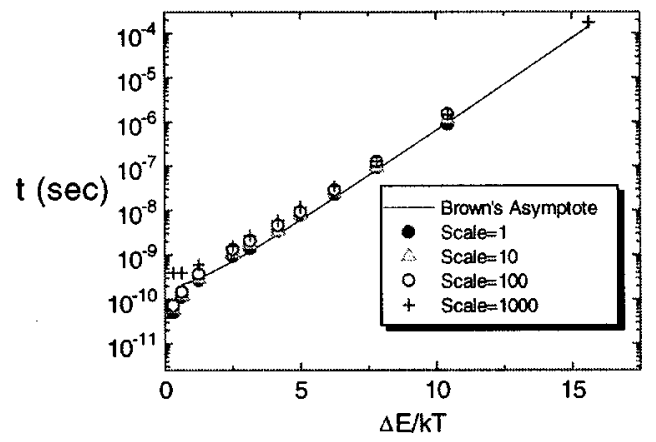

FIG. 2. Comparison of MC relaxation times for different time scales. MC acceptance probability is not taken into account.

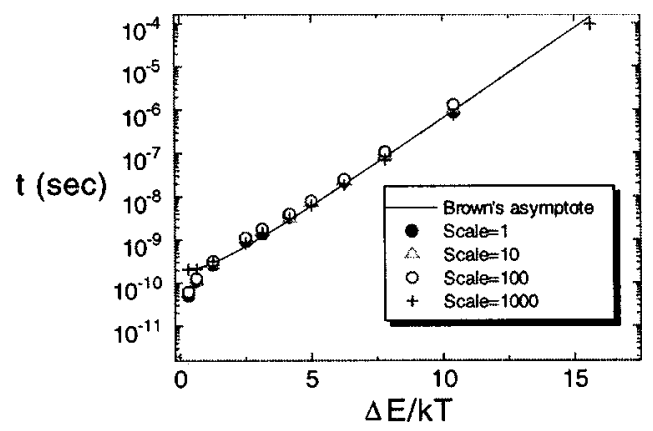

FIG. 3. Comparison of MC relaxation times for different time scales. MC acceptance probability is taken into account.

not affect very much the drastic decrease in computation time for large scales. For instance, in the case of energy barrier equal to 2.5 , the scale $=10$ turned out to be 9.2 times more effective than the scale $=1$. The same characteristic for the scale $=10^{2}$ was 76 , for the scale $=10^{3}-410$, and for the scale $=10^{4}-1100$, respectively. This fact might become an important point for further calculations of relaxation rates over very large energy barriers.

\section{v. CONCLUSIONS}

A new MC approach, which incorporates the information on the size of a real time step, has been used for the calculation of relaxation times of magnetization. Its comparison with corresponding theoretical asymptotic solutions and LD calculations for the ensemble of single-domain particles showed its validity in the considered range of energy barriers. This computational method has also been tested on the implementation of different time scales, which is important for the rising effectiveness of MC calculations. It has been demonstrated that all calculated data are convergent to the expected values if the probability of MC acceptance is explicitly used. It is important to note that the use of large time steps is potentially extremely important for systems of interacting particles where speed requirements are important. The work presented here suggests that increases of speed up to three orders of magnitude relative to the LD technique are possible using the time quantified $\mathrm{MC}$ technique, which represents an important development.

Financial support of the UK EPSRC is acknowledged. R.S.-R. is grateful to the Spanish Ministry of Education for the provision of postdoctoral research grant. The authors thank D. Hinzke for helpful discussions.

${ }^{1}$ W. F. Brown, Phys. Rev. 130, 1677 (1963).

${ }^{2}$ W. T. Coffey, Yu. P. Kalmykov, E. S. Massawe, and J. T. Waldron, J. Chem. Phys. 99, 401 (1993).

${ }^{3}$ R. Smirnov-Rueda, J. D. Hannay, O. Chubykalo, R. W. Chantrell, and J. M. Gonzalez, IEEE Trans. Magn. 35, 3730 (1999).

${ }^{4}$ U. Nowak, R. W. Chantrell, and E. C. Kennedy, Phys. Rev. Lett. (submitted).

${ }^{5}$ D. Hinzke, U. Nowak, and K. D. Usadel, Proceedings SDHS'99 Duisburg (World Scientific, Singapore, 1999).

${ }^{6}$ K. Binder, Monte Carlo Methods in Statistical Physics (Springer, Berlin, 1979).

${ }^{7}$ A. Lyberatos, D. V. Berkov, and R. W. Chantrell, J. Phys.: Condens. Matter 5, 8911 (1993).

${ }^{8}$ W. T. Coffey, D. S. F. Crothers, J. L. Dorman, L. J. Geoghegan, and E. C. Kennedy, Phys. Rev. B 58, 3249 (1998).

Published without author corrections 